\title{
Thestate of the filament
}

\author{
AddekeH. Aguda', LedieD. Burtridk \& RobertC. Robinsan ${ }^{1+}$ \\ ${ }^{1}$ U ppsala University, Uppsala, Sweden, and ${ }^{2}$ The University of British Columbia, Vancouver, British Columbia, Canada
}

\begin{abstract}
Movement is a defining characteristic of life. Macroscopic motion is driven by the dynamic interactions of myosin with actin filaments in muscle. Directed polymerization of actin behind the advancing membrane of a eukaryotic cell generates microscopic movement. D espite the fundamental importance of actin in these processes, the structure of the actin filament remains unknown. The Holmes model of the actin filament was published 15 years ago, and although it has been widely accepted, no high-resolution structural data have yet confirmed its veracity. Here, we review the implications of recently determined structures of F-actin-binding proteins for the structure of the actin filament and suggest a series of in silico tests for actinfilament models. We also review the significance of these structures for the arp2/3-mediated branched filament.

Keywords: actin filament; arp2/3; W H 2; gel solin; structure

EMBO reports(2005) 6, 220-226. doi:10.1038/sj.embor.7400363
\end{abstract}

\section{The structure of the actin monomer}

In this review, we examine the current understanding of the structures of the actin filament and the branched filament that is generated by actin-related protein $2 / 3$ complex (arp2/3), and pose the question, "Do we know the structure of the actin filament?" Before building up these complex actin structures, it is worth asking the seemingly trivial question, "Do we know the structure of the actin monomer?" The answer to this question is surely "Of course we do." After all, there are reports of highresolution structures of actin bound to a myriad of sequestering agents: proteins such as DNase I (Kabsch et al, 1990), profilin (Chik et al, 1996; Schutt et al, 1993), gelsolin (Burtnick et al, 2004; M cLaughlin et al, 1993; Robinson et al, 1999), vitamin-Dbinding protein (DBP; Otterbein et al, 2002), and a hybrid between gelsolin domain 1 and thymosin- $\beta 4$ (Irobi et al, 2004); small molecules such as macrolides (Klenchin et al, 2003); actin crosslinked to itself (Bubb et al, 2002) or rhodamine (O tterbein et al, 2001); and in combination with many binding partners such

\footnotetext{
${ }^{1}$ Department of M edical Biochemistry and M icrobiology, U ppsala Biomedical Center, Uppsala University, U ppsala 751 23, Sweden

2Department of Chemistry and Centrefor Blood Research, TheUniversity

of British Columbia, Vancouver, British ColumbiaV6T 1Z1, Canada

+Corresponding author. Tel: +46 18471 4933; Fax: +46 18471 4975;

E-mail: bob.robinson@imbim.uu.se
}

Submitted 23 N ovember 2004; accepted 25 January 2005 as ciboulot/latrunculin (H ertzog et al, 2004) and the Bnil formin homology domain 2 (FH 2)/rhodamine (O tomo et al, 2005). These actin structures may be classified as 'open' (Fig 1B), as observed in a profilin:actin structure (Chik et al, 1996), or 'closed', as is seen for all others (Fig 1A), including a form of profilin:actin (Schutt et al, 1993). Actin consists of four subdomains that are arranged in pairs (Fig $1 \mathrm{~A}-\mathrm{C}$ ) that sandwich the nucleotide and a metal ion. Minor differences can be seen between closed-form structures, which are most apparent in the conformation of a loop of subdomain 2 (D-loop) and slight variations in the angle between the two halves of actin. In the open form, subdomain 2 rotates away from subdomain 4 to open the nucleotide-binding cleft and leave a clear path for nucleotide exchange. Although the large number of known structures that have the closed conformation clearly suggest it to be the structure of the monomer, we should examine the set of structures in question and consider that most of the agents used to stabilize them also affect nucleotide exchange. W ith the exception of profilin, these agents inhibit nucleotide exchange probably by narrowing the nucleotide-binding cleft, and thus lock actin in the closed form. Therefore, the structure of the actin monomer in isolation is not known with absolute certainty. It may oscillate between the welldefined closed form and more open forms, such as the profilin:actin open structure, in order to exchange nucleotide (that is, move from the structure presented in Fig $1 C$ through to that in Fig $1 B$ and onto the one in Fig $1 \mathrm{~A}$ in order to replace ADP with ATP). Despite uncertainties in the structure of the isolated actin monomer, we know the structures of profilin:actin (Chik et al, 1996; Schutt et al, 1993) and have a good idea of the Wiscott-Aldrich syndrome protein (WASP) homology 2 domain (WH2):actin structure. These are the biologically relevant forms of the actin monomer that are used by cells to feed filament elongation.

\section{Do we know the structure of the actin filament?}

Before addressing this issue, our definition of the term 'structure' should be established. Protein-protein interactions occur on a scale that requires the precise positioning of individual atoms at the interaction interface. Protein crystallography and nuclear magnetic resonance (N M R) can be used to determine structures at such a resolution. M ethods that do not afford the appropriate resolution, or that lack certainty in their interpretation, produce three-dimensional representations that are designated as models rather than structures. 
A

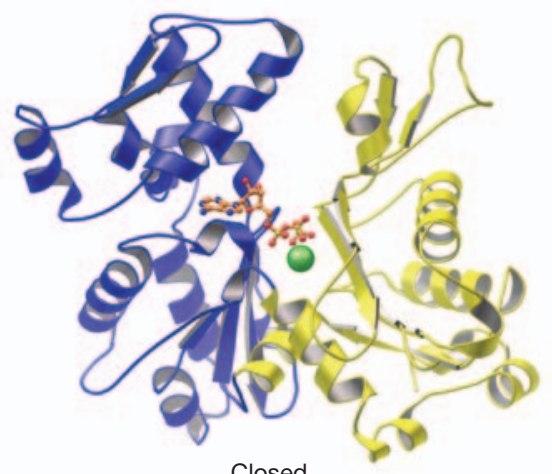

Closed

D

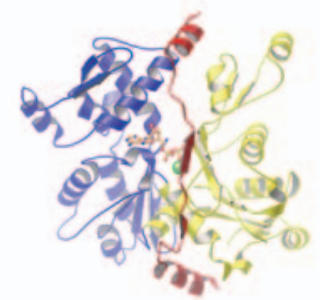

WH2:actin

F

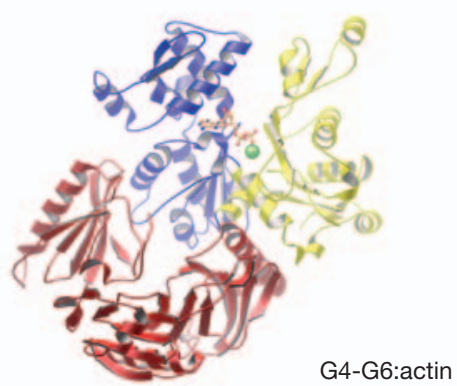

B

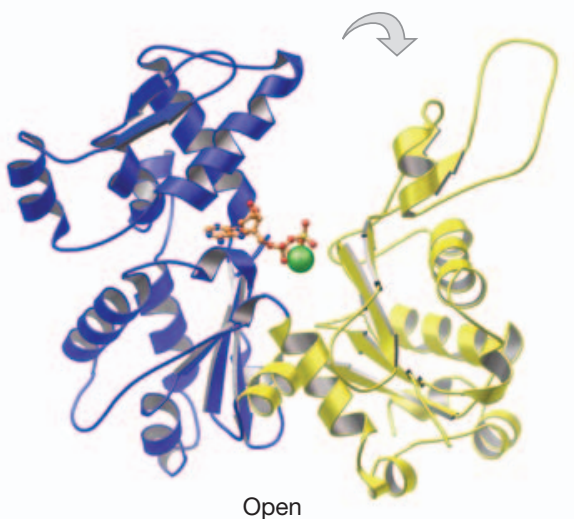

E

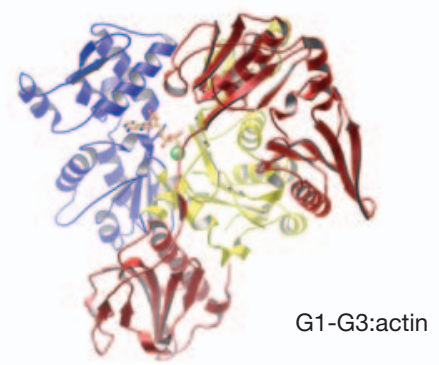

G

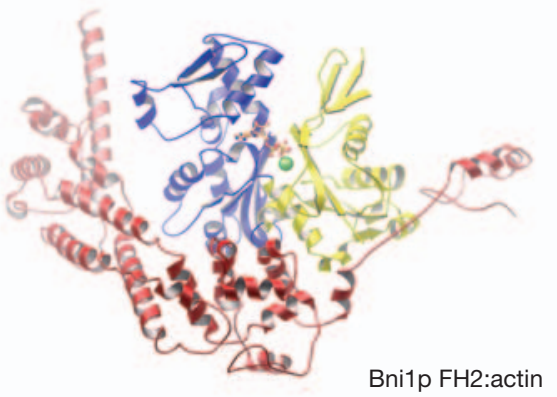

C

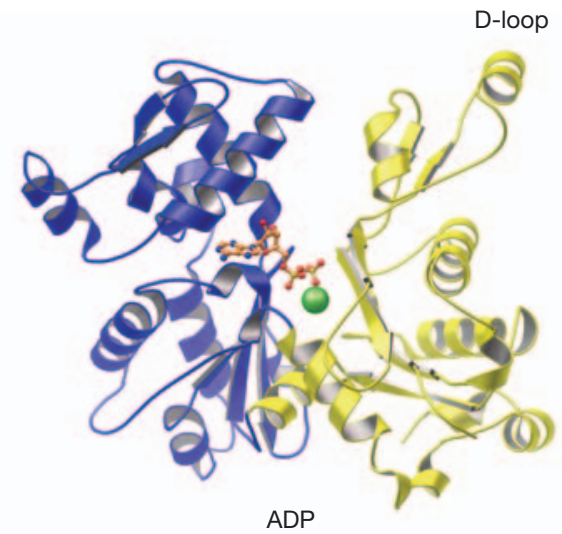

H

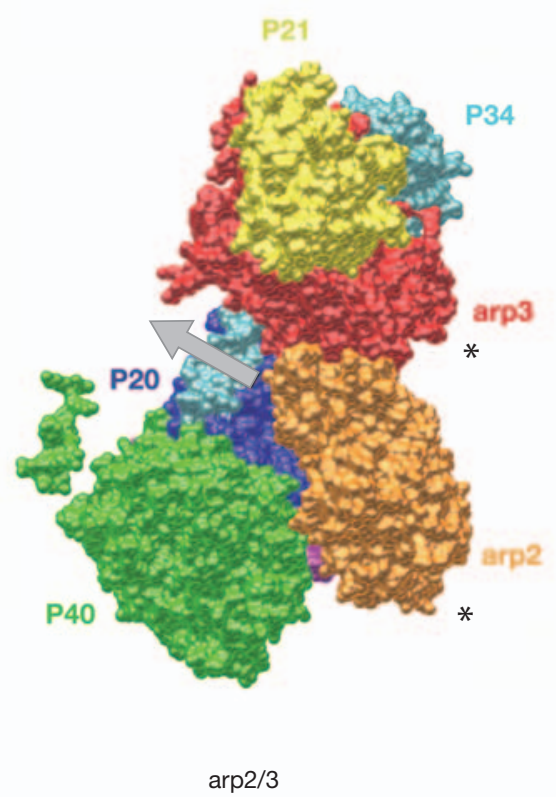

Fig1|Actin and actin-related protein (arp) structures. (A) An example of the closed form of the actin monomer (Protein DataBase (PDB) code 2BTF). The nucleotide (ATP) is shown as a ball-and-stick representation and the metal ion as a green sphere. The subdomains are labelled 1-4 and are coloured yellow $(1,2)$ and blue (3,4). (B) Theopen form of the actin monomer (PDB code $1 \mathrm{HLU}$ ). (C) A second example of the closed structure of the actin monomer (PDB code 1] 6Z). In this ADP-bound structure the distinctiveD-loop is labelled. (D) A composite of Wiscott-Aldrich syndrome protein homology 2 (WH2) motif structures (red) in complex with actin (PDB codes 1T44 and 1SQK). (E) TheN-terminal half of gelsolin (G1-G3, red) bound to actin (PDB code 1RGI). (F) The C-terminal half of gelsolin (G4-G6, red) bound to actin (PDB code 1H 1V). (G) The Bnil formin homology domain 2 (FH2) domain bound to actin (PDB code 1Y64). Only one-half of the FH 2 dimer is depicted for clarity. (H) Thearp2/3 complex (PDB code 1K8K). Arp2 (orange), arp3 (red), P40 (green), P34 (cyan), P21 (yellow) and P20 (blue) are visible in this orientation, while P16 (purple) is obscured from view. The missing portion of arp2 is modelled (Robinson etal, 2001). Asterisks represent the barbed ends of arp2 and arp3. An arrow indicates the direction in which arp2 is required to move to adopt a Holmes-likefilament orientation with respect to arp3.

These include electron-diffraction models, fibre-diffraction models and models based on crystallographic contacts. So, the answer is "No", we do not know the structure of the actin filament, but we do have several models. These models include the Holmes fibrediffraction model (Holmes closed model; Holmes et al, 1990); the
Egelman electron microscopy reconstruction model (Belmont et al, 1999), which is a Holmes-like model that comprises open-cleft actin protomers (Holmes-like open model); and the unrelated ribbon-tohelix hypothesis that is based on actin:actin crystal contacts within profilin:actin crystals (Schutt et al, 1993). 
Given that we do not know the structure of the filament, can the database of known actin monomer structures be used to distinguish the best filament models? Recently, several actin monomer structures have been determined involving proteins that are also able to interact with actin filaments. Some members of the WH 2 family of proteins release their bound actin monomer during polymerization, and at least one, ciboulot, is able to cap the pointed end of the filament (Fig 1D; Hertzog et al, 2004; Irobi et al, 2004). Gelsolin is able to cap, sever and nucleate actin filaments and, under in vitro conditions, form complexes with actin monomers (Fig 1E,F; Burtnick et al, 2004; Robinson et al, 1999). $\mathrm{Bni1} \mathrm{FH} 2$ is able to nucleate and processively elongate actin filaments (Fig 1G; O tomo et al, 2005). Arp2/3 is a complex of seven proteins that includes two subunits, arp2 and arp3, each of which resembles actin in structure (Fig $1 \mathrm{H}$; Robinson et al, 2001). The two arps are thought to adopt a filament-like orientation in order to act as the first two subunits in the arp2/3 branched daughter filament. If we assume that the interactions of these filamentbinding proteins with actin, or with the arps, will be similar to a first approximation, whether they are bound to a monomer or to a filament, these structures have relevance in the evaluation of actin filament models. However, it should be noted that at least two conformational changes are expected in the actin monomer structure on incorporation into a filament. The first, which occurs immediately on addition of actin monomers to filaments, is evinced by the release of profilin and the increased rate of ATP hydrolysis (Blanchoin \& Pollard, 2002). The second, which follows hydrolysis of ATP and the release of phosphate, is seen by the preference of many filament-binding proteins for filaments that bear a particular nucleotide. Furthermore, many actin-binding proteins, including gelsolin, are known to cause conformational changes in the filament (O rlova et al, 1995). Hence, in the analyses below, we allow moderate conformational changes in the actin-protomer structure and also permit small changes in the protomer orientation.

\section{Interactions across the filament}

Gelsolin, ciboulot and arp2/3 are able to cap actin filaments, and gelsolin, Bni1 FH 2 and arp2/3 can nucleate filaments (Burtnick et al, 2001; Dayel \& M ullins, 2004; H ertzog et al, 2002; 0 tomo et al 2005). Therefore, the actin-monomer-bound forms of ciboulot, gelsolin and FH2 must allow for the formation of actin:actin contacts across the filament, while obscuring the appropriate filament end. Similarly, within the arp2/3 complex, the interactions of the accessory proteins with arp2 and arp3 should allow arp2 and arp3 to adopt an orientation appropriate to an actin filament. Superposition of the actin structures from the G1-G3, G4-G6, $\mathrm{FH} 2$ and the $\mathrm{WH} 2$ domain actin complexes, and overlaying arp2 and arp3 onto actin with their accessory proteins P40, P16 and $P 20$, and P21 and P34, respectively, provides a picture of the external face of an actin filament (Fig 2A-D). These filament-binding proteins extensively cover one face and the two sides of an actin protomer, while leaving its opposite face free. The unobscured face represents the surface that is available for actin-actin interactions across the filament and provides the first test of validity for the filament models. In the Holmes-like models, the actin-actin interaction footprint is buried in the cross-filament interactions (Fig 2D), which satisfies this test. By default, models that are grossly different to the Holmes model are contrary to this analysis.

\section{Biology of the interacting proteins}

Relations between the structures and biology of the F-actin-binding proteins that are discussed above provide further tests for models of the actin filament. The WH2 motif superfamily includes the $\beta$ thymosin subfamily, members of which sequester actin monomers and do not directly release them for filament elongation. The WH2 domain structures show that the two termini of thymosin- $\beta 4$ are able to hinder its inclusion at either the barbed or the pointed end of a Holmes-like filament (Hertzog et al, 2004; Irobi et al, 2004). Two modifications exist that turn the sequestering $\beta$-thymosin into the more general WH2 barbed-end elongating module. First, the carboxyl terminus is shortened or its actin binding is impaired to allow inclusion of W H2:actin at the barbed end of the filament (H ertog et al, 2004). Second, proteins that contain many W H 2 motifs are consistent with decorating neighbouring actin subunits on one face of the Holmes-like models, and during elongation may deliver actin monomers to the barbed end (Irobi et al, 2004).

When the structures of the two halves of gelsolin bound to actin are superimposed onto the Holmes closed model of the filament, steric clashes occur that involve gelsolin domains $\mathrm{G} 1$ and $\mathrm{G} 4$ with the neighbouring longitudinally associated actin protomers, in line with the capping function of these domains (Burtnick et al, 2004). Furthermore, G2, the F-actin-binding domain, is orientated with respect to a third actin to allow their predicted binding surfaces to come together (Choe et al, 2002; Puius et al, 2000). Hence, the $\mathrm{H}$ olmes-like models show no disparity with the gel solin structures.

The Bni1 FH2:actin structure provides a powerful indication as to the mechanisms of nucleation, capping and elongation by the $\mathrm{FH} 2$ domain from the formin family of proteins ( $\mathrm{O}$ tomo et al, 2005). Each FH 2 chain contacts three actin monomers to create a filament nucleus. The conformation of the $\mathrm{FH} 2$ domain in the crystal prevents addition of a Holmes-like barbed-end actin through steric clashes. However, domain swapping by $\mathrm{FH} 2$ suggests how this steric hindrance may be overcome to allow processive elongation. Indeed, the crystals contain a pseudo$\mathrm{H}$ olmes-like filament that is decorated by an $\mathrm{FH} 2$ polymer formed through domain swapping.

Finally, the structure of the arp2/3 complex shows that the relative positions of arp2 and arp3 would allow them to be moved into a Holmes-like orientation without disrupting the integrity of the remaining five subunits (Figs $1 \mathrm{H}, 3 \mathrm{~A}$, B; Robinson et al, 2001). Closer inspection reveals that the D-loop of arp2 would clash with P21 if an ADPactin-like conformation were adopted by arp2 (Fig 1C). This clash does not occur if arp2 adopts an ATP-actin-like conformation. The structure of the ATP-bound closed form of arp3 (within arp2/3) was recently determined, and it is reasonable to assume that arp2 will also adopt an ATP-bound closed form during activation (N olen et al, 2004). Hence, the arp2/3 structure provides a stern test for the Holmes-like models.

Given that the biological activity of the filament-binding proteins can be superficially accounted for by a filament model that is composed of the actin monomer structure, we expect that only subtle changes in the actin protomer structure will occur on polymerization.

\section{H olmes-like models: open versus closed protomers}

The Holmes closed model and the Holmes-like open model conflict in one regard: namely, the width of the nucleotide-binding cleft (Belmont et al, 1999; Holmes et al, 1990). The high-resolution structures under consideration here provide data relevant to this controversy. First, thymosin- $\beta 4$ interacts with an actin monomer by 

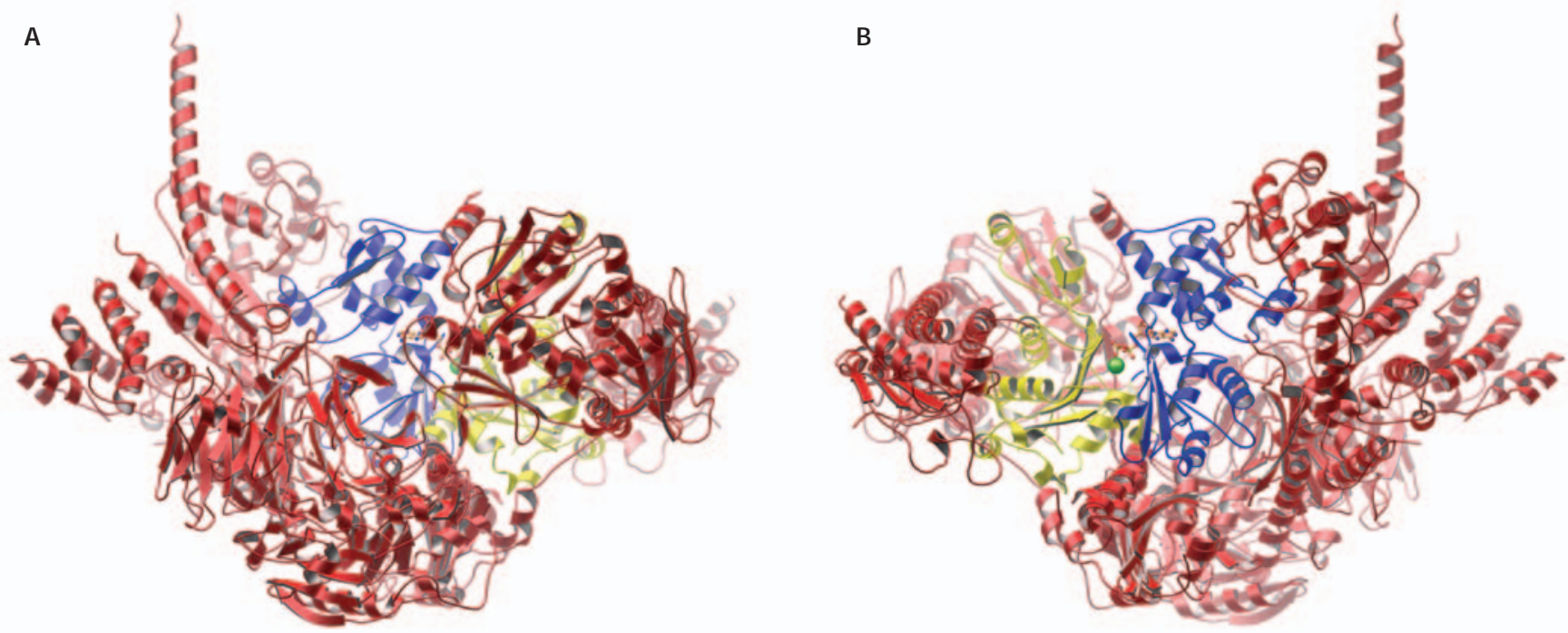

C

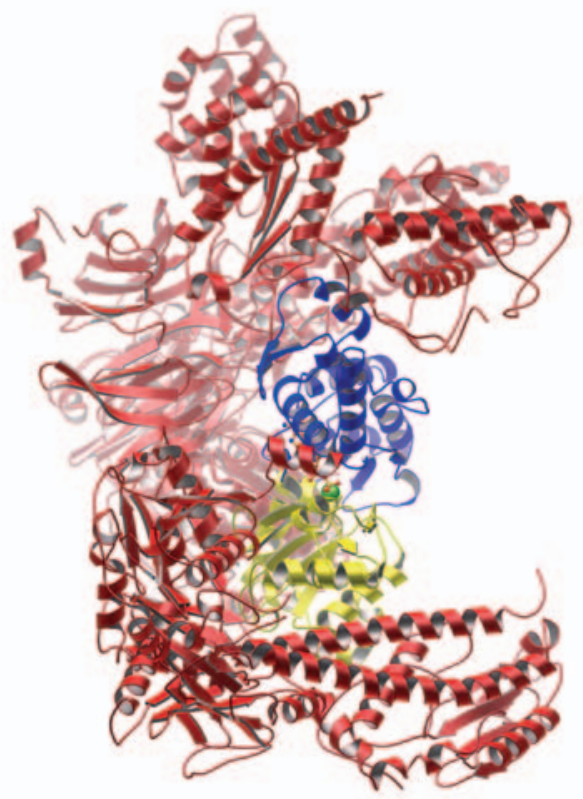

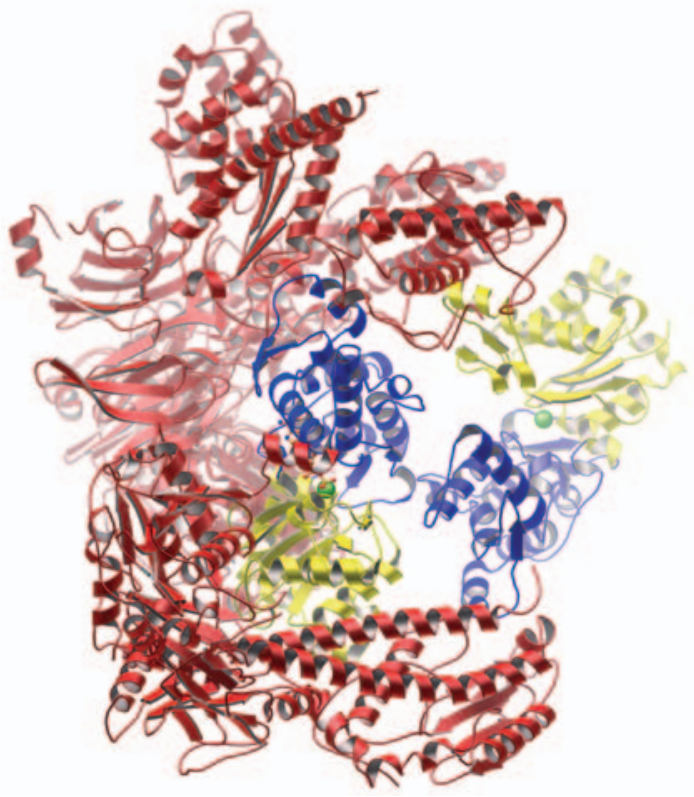

Fig2 | The actin-actin interaction footprint. (A) Superposition of arp2 (with its accessory proteins P40, P20 and P16), arp3 (with P34 and P21) and the actin complexes from Fig $1 D$ - G onto a single actin monomer. The binding proteins are shown in red and actin is presented in the sameorientation as in Fig $1 \mathrm{~A}$. This face of actin is almost completely obscured by the binding proteins. (B) A $180^{\circ}$ rotation of (A) around they-axis. This face of actin is free of binding proteins and available for actin-actin interactions. (C) Rotation of (A) to a position looking down onto the nucleotide binding cleft (the pointed end). (D) The sameas (C), but with a second actin from the Holmes model of the actin filament (Holmes etal, 1990). The Holmes orientated actin is located at the actin-actin interaction footprint.

inserting its C-terminal helix between actin subdomains 2 and 4 . This binding surface would be abolished in the open form of actin. Hence, for related WH 2 proteins to cap the filament through this interaction, the terminal pointed-end actin protomer is required to be in a closed conformation. Second, gelsolin caps the barbed end of a filament and secures the gelsolin-bound actins in a closed conformation by inserting a helix between actin subdomains 1 and 3 , thereby preventing actin from adopting an open form. The F-actinbinding domain ( $\mathrm{G} 2$ ) also requires a closed conformation in the terminal actin to allow it access to its binding site on the longitudinally adjacent actin (Burtnick et al, 2004). Thus, both ends of capped filaments require actin protomers that adopt a closed conformation. Furthermore, this argument may be extended to the centre of the filament. In order for gelsolin to bind to the side of a filament and initiate severing, G2 must bind to two actin protomers, one of which must be in the closed conformation to provide the correct binding surface for G2. Therefore, these structures support Holmes-like models that include at least a proportion of closed actin protomers. 
A

B
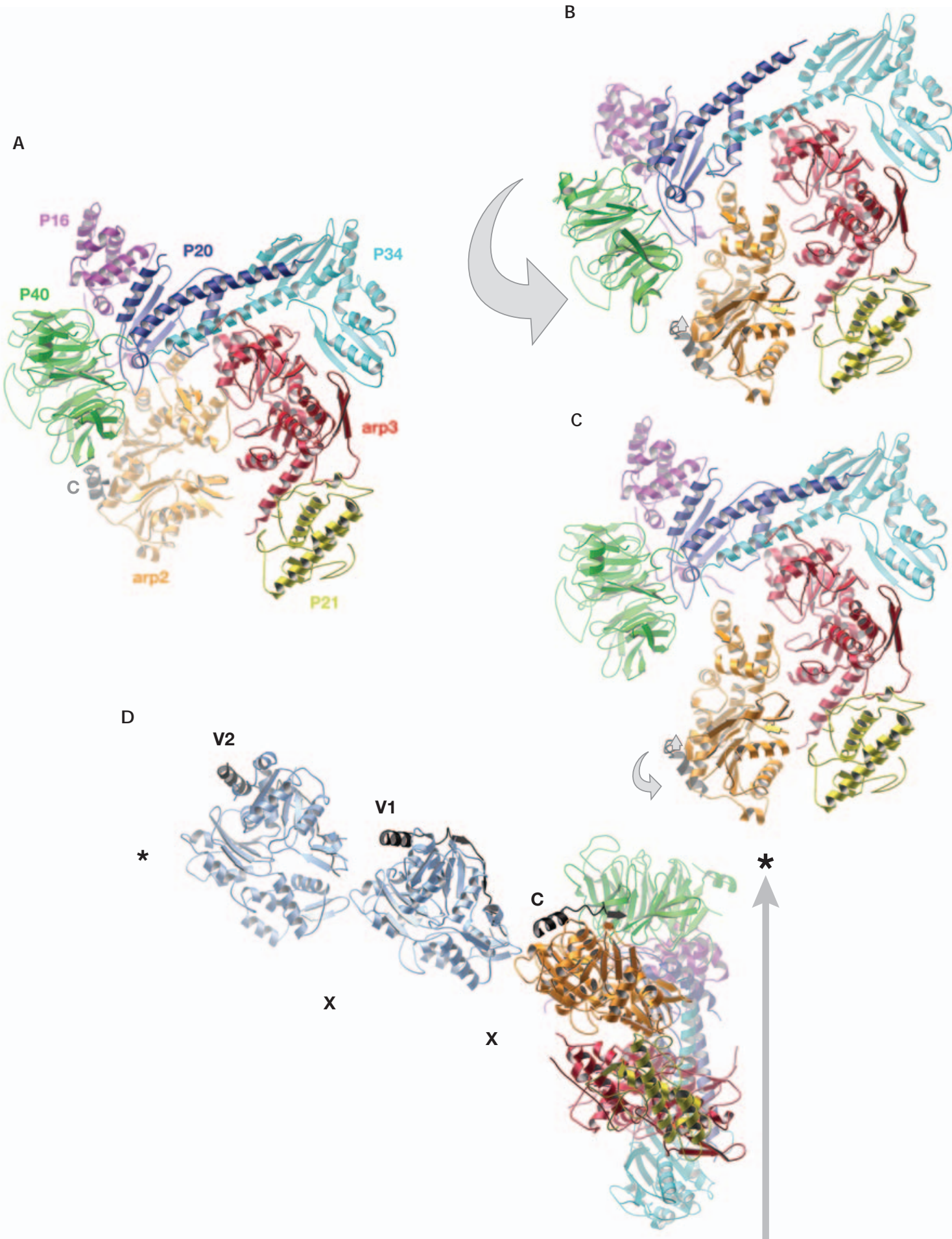

Fig3| M odels of arp2/3 conformational changes and branching. (A) M odel of arp2/3 with bound C-motif ( grey, labelled C). The C-motif was modelled by positioning the $\mathrm{N}$-terminal half of aWH 2 motif onto arp2 in the same manner in which it interacts with actin (Irobi etal, 2004). (B) Rotation model. Arp2/P40/P16/P20 have been rotated relativeto arp3/P34/P21 to allow arp2 to adopt a H olmes filament orientation relativeto arp3. (C) Arp2 migration model. Arp2 has been moved into a H olmes filament orientation relative to arp3. (D) M odel of an arp2/3-mediated branch. Thearp2/3 model from (C) has been rotated and two actin:WH 2 motif structures docked onto arp2 in a H olmes filament orientation. The two WH 2 motifs, as found in N-WASP are labelled V 1 and $\mathrm{V} 2$, and the $\mathrm{C}$ region is indicated. Thearrow suggests the flat surface to which the mother filament may bind, creating the $70^{\circ}$ branch. Asterisks denotethe barbed end of the filaments and ' $x$ ' marks the positions of joining actin monomers. 


\section{The arp2/3 branched filament}

The recent structures of the W H 2-motif:actin complexes have provided new insights into the activating proteins for the arp2/3mediated branch. The arp $2 / 3$ activators- the WASP family of proteins - contain a minimal activating motif, VCA, that interacts both with actin ( $\mathrm{V}$, a WH2 motif) and arp2/3 (CA; Panchal et al, 2003). The structures of ciboulot and thymosin- $\beta 4$ define the portion of VCA that contacts an actin monomer (Hertog et al, 2004; Irobi et al, 2004). In the double-W H 2-motif-containing protein N WASP, the proximity of the two $\mathrm{V}$ motifs limits them to assembly with two longitudinally related actin protomers during nucleation. The structures of ciboulot and thymosin- $\beta 4$ also led both sets of authors to predict that the amphipathic helix in the $C$ region of the WASP family of proteins will interact with arp2 in a manner similar to that between the amino-terminal helix of the $\mathrm{WH} 2$ motifs and actin. The implication, again due to proximity considerations, is that the VCA motif will dock theV-bound actin onto arp2. This idea is corroborated by the activity of a hybrid arp2/3 in which arp2 was fused to a V motif; the hybrid protein supported branch formation in the presence of CA (G oley et al, 2004).

The arp2/3 binding sites for CA are widespread and are found on arp2, arp3, P40 and P21 (Zalevsky et al, 2001). Recently, recombinant P40 was shown to contain a high-affinity binding site for VCA that is roughly equivalent to that of the entire arp2/3 complex. This contact includes P40 interacting with the A region (Pan et al, 2004), which was previously shown to bind to arp3 and P21 (Machesky \& Insall, 1998; Weaver et al, 2002). Given the proposed docking of the V-bound actin onto arp2 and the high-affinity interaction of CA with $\mathrm{P} 40$, it is difficult to reconcile the distances between arp2/P40 and arp3/P21 with the chain length of CA (the length of the amphipathic helix +24 residues in the case of WASP-family verprolin homologous protein 1 (WAVE1)). Conformational change in arp2/3 provides at least a partial explanation (Goley et al, 2004). However, a dynamic role for CA may also be considered, in which CA may destabilize the inactive conformation through binding to one site on arp2/3 (for example, P40) and initiate a conformational change. The active conformation of arp2/3 may then offer a competing CA-binding site (perhaps arp3/P21) that would stabilize active arp2/3 once occupied.

Two structural rearrangements of arp2/3 have been suggested that will bring arp2 and arp3 into a H olmes-like alignment in order to provide the nucleus for the daughter filament. The rotation model predicts that the two halves of arp2/3 (arp3/P21 versus arp2/P16/P40; Robinson et al, 2001) will rotate relative to each other on the backbone of P20/P34 (Fig 3A,B). Alternatively, the arp2-migration model suggests that interaction between P40 and arp2 is released, which would allow arp2 to be delivered to arp3 by the $\mathrm{N}$-terminal extension of P16 (Fig 3A,C; Irobi et al, 2004). Either mechanism allows for the construction of models of the arp2/3 branch solely through structural superposition of known structures and the Holmes model (Fig 3D). The flat face of the arp2/3 complex makes a natural angle of about $70^{\circ}$ with the daughter branch, which suggests the position of the mother filament.

In conclusion, recent structures of F-actin-binding proteins have allowed us to reassess filament models. These structures are consistent with a Holmes-like filament that comprises, at least in part, closed-cleft protomers. The structures of arp $2 / 3$ and the WH 2 motif in complex with actin have led to possibilities for the construction of the arp2/3 branched filament. However, these predictions are only models and more structural data are required to determine their worth.

\section{ACKN O W LED G EM ENTS}

TheEM BO Young Investigator schemeand the Swedish M edical Research Council support R.C.R. L.B. thanks the Heart and Stroke Foundation of British Columbia and Yukon for support.

\section{REFEREN CES}

Belmont LD, O rlova A, D rubin DG, Egelman EH (1999) A change in actin conformation associated with filament instability after Pi release. Proc N atl Acad Sci USA 96: 29-34

Blanchoin L, Pollard TD (2002) Hydrolysis of ATP by polymerized actin depends on the bound divalent cation but not profilin. Biochemistry 41: 597-602

Bubb M R, Govindasamy L, Yarmola EG, Vorobiev SM, Almo SC, Somasundaram T, Chapman MS, Agbandje-M CKenna M, McKenna R (2002) Polylysine induces an antiparallel actin dimer that nucleates filament assembly: crystal structure at 3.5- $\AA$ resolution. J Biol Chem 277: 20999-21006

Burtnick LD, Robinson RC, Choe S (2001) Structure and function of gelsolin. Results Probl Cell Differ 32: 201-211

Burtnick LD, U rosev D, Irobi E, N arayan K, Robinson RC (2004) Structure of the $\mathrm{N}$-terminal half of gelsolin bound to actin: roles in severing, apoptosis and FAF. EM BO | 23: 2713-2722

Chik JK, Lindberg U, Schutt CE (1996) The structure of an open state of $\beta$-actin at 2.65 A resolution. J M ol Biol 263: 607-623

Choe H, Burtnick LD, M ejillano M, Yin HL, Robinson RC, Choe S (2002)The calcium activation of gelsolin: insights from the $3 \AA$ structure of the G4-G6/actin complex. J Mol Biol 324: 691-702

Dayel MJ, Mullins RD (2004) Activation of Arp2/3 complex: addition of the first subunit of the new filament by a WASP protein triggers rapid ATP hydrolysis on Arp2. PLoS Biol 2: E91

Goley ED, Rodenbusch SE, Martin AC, Welch MD (2004) Critical conformational changes in the arp2/3 complex are induced by nucleotide and nucleation promoting factor. Mol Cell 16: 269-279

Hertzog M, Yarmola EG, Didry D, Bubb M R, Carlier M F (2002) Control of actin dynamics by proteins made of $\beta$-thymosin repeats: the actobindin family. J Biol Chem 277: 14786-14792

Hertzog M et al (2004) The b-thymosin/W H 2 domain; structural basis for the switch from inhibition to promotion of actin assembly. Cell 117: 611-623

Holmes KC, Popp D, Gebhard W, Kabsch W (1990) Atomic model of the actin filament. N ature 347: 44-49

Irobi E, Aguda AH, Larsson M, Guerin C, Yin HL, Burtnick LD, Blanchoin L, Robinson RC (2004) Structural basis of actin sequestration by thymosinB4: implications for W H 2 proteins. EM BO J 23: 3599-3608

Kabsch W, M annherz HG, Suck D, Pai EF, Holmes KC (1990) Atomic structure of the actin:D N ase I complex. N ature 347: 37-44

Klenchin VA, Allingham JS, King R, Tanaka J, M arriott G, Rayment I (2003) Trisoxazole macrolide toxins mimic the binding of actin-capping proteins to actin. N at Struct Biol 10: 1058-1063

Machesky LM, Insall RH (1998) Scarl and the related Wiskott-Aldrich syndrome protein, WASP, regulate the actin cytoskeleton through the Arp2/3 complex. Curr Biol 8: 1347-1356

McLaughlin PJ, Gooch JT, M annherz HG, Weeds AG (1993) Structure of gelsolin segment 1-actin complex and the mechanism of filament severing. Nature 364: 685-692

N olen BJ, Littlefield RS, Pollard TD (2004) Crystal structures of actin-related protein 2/3 complex with bound ATP or ADP. Proc N atl Acad Sci U SA 101: 15627-15632

O rlova A, Prochniewicz E, Egelman EH (1995) Structural dynamics of F-actin. II. Cooperativity in structural transitions. J Mol Biol 245: 598-607

O tomo T, Tomchick DR, O tomo C, Panchal SC, Machius M, Rosen M K (2005) Structural basis of actin filament nucleation and processive capping by a formin homology 2 domain. N ature 433: 488-494

O tterbein LR, G raceffa P, D ominguez R (2001)The crystal structure of uncomplexed actin in the ADP state. Science 293: 708-711

O tterbein LR, Cosio C, G raceffa P, D ominguez R (2002) Crystal structures of the vitamin $D$-binding protein and its complex with actin: structural basis of the actin-scavenger system. Proc N atl Acad Sci USA 99: 8003-8008 
Pan F, Egile C, Lipkin T, Li R (2004) ARPC1/Arc40 mediates the interaction of the actin-related protein 2 and 3 complex with Wiscott-Aldrich syndrome protein family activators. J Biol Chem 279: 54629-54636

Panchal SC, Kaiser DA, Torres E, Pollard TD, Rosen M K (2003) A conserved amphipathic helix in WASP/Scar proteins is essential for activation of Arp2/3 complex. Nat Struct Biol 10: 591-598

Puius YA, Fedorov EV, Eichinger L, Schleicher M, Almo SC (2000) M apping the functional surface of domain 2 in the gelsolin superfamily. Biochemistry 39: 5322-5331

Robinson RC, M ejillano M, LeVP, Burtnick LD, Yin HL, Choe S (1999) D omain movement in gelsolin: a calcium-activated switch. Science 286: 1939-1942

Robinson RC, Turbedsky K, Kaiser DA, M archand JB, Higgs H N, Choe S, Pollard TD (2001) Crystal structure of Arp2/3 complex. Science 294: 1679-1684

Schutt CE, M yslik JC, Rozycki M D, G oonesekere N C, Lindberg U (1993) The structure of crystalline profilin- $\beta$-actin. $N$ ature 365: 810-816

Weaver AM, Heuser JE, Karginov AV, Lee W L, Parsons JT, Cooper JA (2002) Interaction of cortactin and N-WASp with Arp2/3 complex. Curr Biol 12: $1270-1278$

Zalevsky J, Lempert L, Kranitz H, Mullins RD (2001) Different WASP family proteins stimulate different Arp2/3 complex-dependent actinnucleating activities. Curr Biol 11: 1903-1913

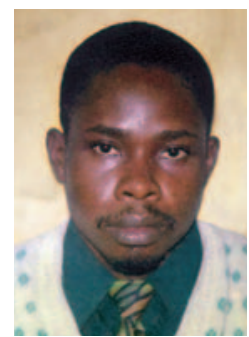

AddekeH.Aguda

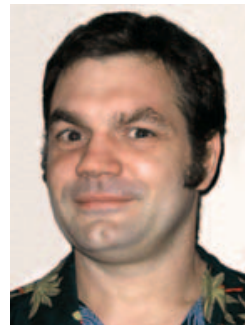

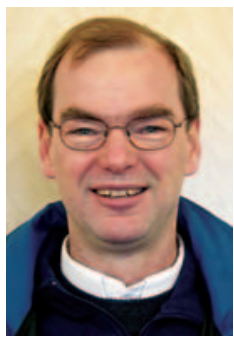

LedieD. Burtridk

RobertC. Robinsonisthe redipient of anEMBO

Younglnvestigator Award 